\title{
Detection of miR-155-5p and imaging lung cancer for early diagnosis: in vitro and in vivo study
}

\author{
Hai-Zhen Zhu ${ }^{1}$. Chun-Ju Fang ${ }^{1} \cdot$ Yi Guo $^{2}$ - Qi Zhang ${ }^{1} \cdot$ Li-Min Huang $^{1}$ - Dong Qiu ${ }^{1}$. Guang-Peng Chen ${ }^{3}$. \\ Xiu-Feng Pang ${ }^{4}$. Jian-Jun $\mathrm{Hu}^{5}$. Jian-Guo Sun ${ }^{3}$. Zheng-Tang Chen ${ }^{3}$ (1)
}

Received: 10 December 2019 / Accepted: 4 May 2020 / Published online: 23 May 2020

(c) The Author(s) 2020

\begin{abstract}
Purpose Currently, the routine screening program has insufficient capacity for the early diagnosis of lung cancer. Therefore, a type of chitosan-molecular beacon (CS-MB) probe was developed to recognize the miR-155-5p and image the lung cancer cells for the early diagnosis.

Methods Based on the molecular beacon (MB) technology and nanotechnology, the CS-MB probe was synthesized selfassembly. There are four types of cells-three kinds of animal models and one type of histopathological sections of human lung cancer were utilized as models, including A549, SPC-A1, H446 lung cancer cells, tumor-initiating cells (TICs), subcutaneous and lung xenografts mice, and lox-stop-lox(LSL) K-ras G12D transgenic mice. The transgenic mice dynamically displayed the process from normal lung tissues to atypical hyperplasia, adenoma, carcinoma in situ, and adenocarcinoma. The different miR-155-5p expression levels in these cells and models were measured by quantitative real-time polymerase chain reaction (qRT-PCR). The CS-MB probe was used to recognize the miR-155-5p and image the lung cancer cells by confocal microscopy in vitro and by living imaging system in vivo.

Results The CS-MB probe could be used to recognize the miR-155-5p and image the lung cancer cells significantly in these cells and models. The fluorescence intensity trends detected by the CS-MB probe were similar to the expression levels trends of miR-155 tested by qRT-PCR. Moreover, the fluorescence intensity showed an increasing trend with the tumor progression in the transgenic mice model, and the occurrence and development of lung cancer were dynamically monitored by the differen fluorescence intensity. In addition, the miR-155-5p in human lung cancer tissues could be detected by the miR-155-5p MB. Conclusion Both in vivo and in vitro experiments demonstrated that the CS-MB probe could be utilized to recognize the miR-155-5p and image the lung cancer cells. It provided a novel experimental and theoretical basis for the early diagnosis of the disease. Also, the histopathological sections of human lung cancer research laid the foundation for subsequent preclinical studies. In addition, different MBs could be designed to detect other miRNAs for the early diagnosis of other tumors.
\end{abstract}

Keywords Lung cancer $\cdot$ MicroRNA $\cdot$ Tumor-initiating cell $\cdot$ Molecular beacon $\cdot$ Chitosan $\cdot$ Molecular imaging

Hai-Zhen Zhu, Chun-Ju Fang, Yi Guo and Qi Zhang have contributed equally to this work.

Jian-Guo Sun

sunjg09@aliyun.com

$\triangle$ Zheng-Tang Chen

chenzhengtang123@163.com

1 Department of Oncology, Guizhou Provincial People's Hospital, Guizhou Cancer Center, Guiyang 550002, China

2 Department of Basic Knowledge, Guiyang Nursing Vocational College, Guiyang 400037, China
3 Cancer Institute of PLA, Xinqiao Hospital, Army Medical University, Chongqing 400037, China

4 Shanghai Key Laboratory of Regulatory Biology, Institute of Biomedical Sciences and School of Life Sciences, East China Normal University, Shanghai 200241, China

5 Department of Pathology, Guizhou Provincial People's Hospital, Guiyang 550002, China 


\section{Introduction}

Owing to late-stage detection and poor treatment, lung cancer is the leading cause of cancer-related deaths worldwide (Wang et al. 2019a, b). The high mortality of lung cancer is always closely associated with late diagnosis (Zhang et al. 2019a, b). Thus, improving the early diagnosis efficiency is very important for the prognosis of patients with lung cancer. However, currently, specific and sensitive molecular targets for the early diagnosis of lung cancer are lacking (OST et al. 2014; Lipińska et al. 2019).

Tumor-initiating cells (TICs) are considered as the source of tumor occurrence, development, recurrence, and metastasis (De et al. 2018; Moro et al. 2015; Yu et al. 2016), thereby finding the TICs may be a novel breakthrough for the early diagnosis of cancers. If the TICs of lung cancer can be recognized and imaged directly based on the fluorescent substances, then the early diagnosis can be implemented. Thus, imaging of cancer cells is currently an active area of research (Lingeshwar Reddy et al. 2018). Previous studies have demonstrated that microRNAs (miRNAs, miR) extensively participate in the occurrence, development, and metastasis of cancers (Tutar et al. 2018). miRNAs are short, highly conserved, noncoding RNAs, 18-22 nucleotides in length, and can function as oncogenes or tumor suppressor genes. Thus, recognition and imaging of miRNAs related to lung cancer cells or TICs might constitute a critical strategy for the early diagnosis of the disease. Among these, miR-155 plays a major role in the occurrence, development, and diagnosis of lung cancer (Zhang et al. 2018; Shao et al. 2019).

Molecular beacon (MB) is a stem loop-structured (hairpin) oligonucleotide that has a reporter fluorophore and a quencher. MB cannot produce fluorescence; however, it hybridizes with the target DNA, RNA, or miRNA to produce fluorescence signals. Therefore, real-time imaging and quantitative analysis of target DNA, RNA, and miRNA can be implemented by detecting the fluorescence signal and intensity (Zhang et al. 2017; Moon et al. 2019; Kang et al. 2011). Since MB is an oligonucleotide, it cannot traverse the cell membrane easily due to the negative surface charge, and is degraded by endogenous nucleases, and, hence, cannot enter the cells directly but only when delivered by an ideal carrier (Kim et al. 2015; Dong et al. 2011). Chitosan (CS) is a natural cationic polysaccharide and has been widely used to mediate the gene transfection of plasmids and small interfering RNAs (siRNAs) (Cao et al. 2019; Rahmani et al. 2015; Jaiswal et al. 2019). In a previous study (Zhu et al. 2014), we used CS nanoparticles combined with MB for the detection and imaging miR$155-5 p$ in non-small cells lung cancer.

The occurrence of lung adenocarcinoma of lox-stop-lox (LSL) K-ras G12D transgenic mice can be induced by instilling Cre adenovirus into the nasal cavity after adulthood. The transgenic mice model displays the dynamic process from normal lung tissues to atypical hyperplasia, adenoma, carcinoma in situ, and adenocarcinoma, and simulates the occurrence and development of human lung adenocarcinoma (DuPage et al. 2009; Sutherland et al. 2014), thereby serving as a preferred animal model for the early diagnosis of lung cancer. Therefore, in the present study, nanotechnology and MB technology were combined, and lung cancer cells, TICs, subcutaneous and lung xenografts in nude mice, and transgenic mice were used as the study subjects to investigate the feasibility of CS nanoparticles (as miR-155-5p MB carrier) in recognizing and imaging miR-155-5p in lung cancer cells (Fig. 1). This strategy would provide a novel technology for the early diagnosis of lung cancer.

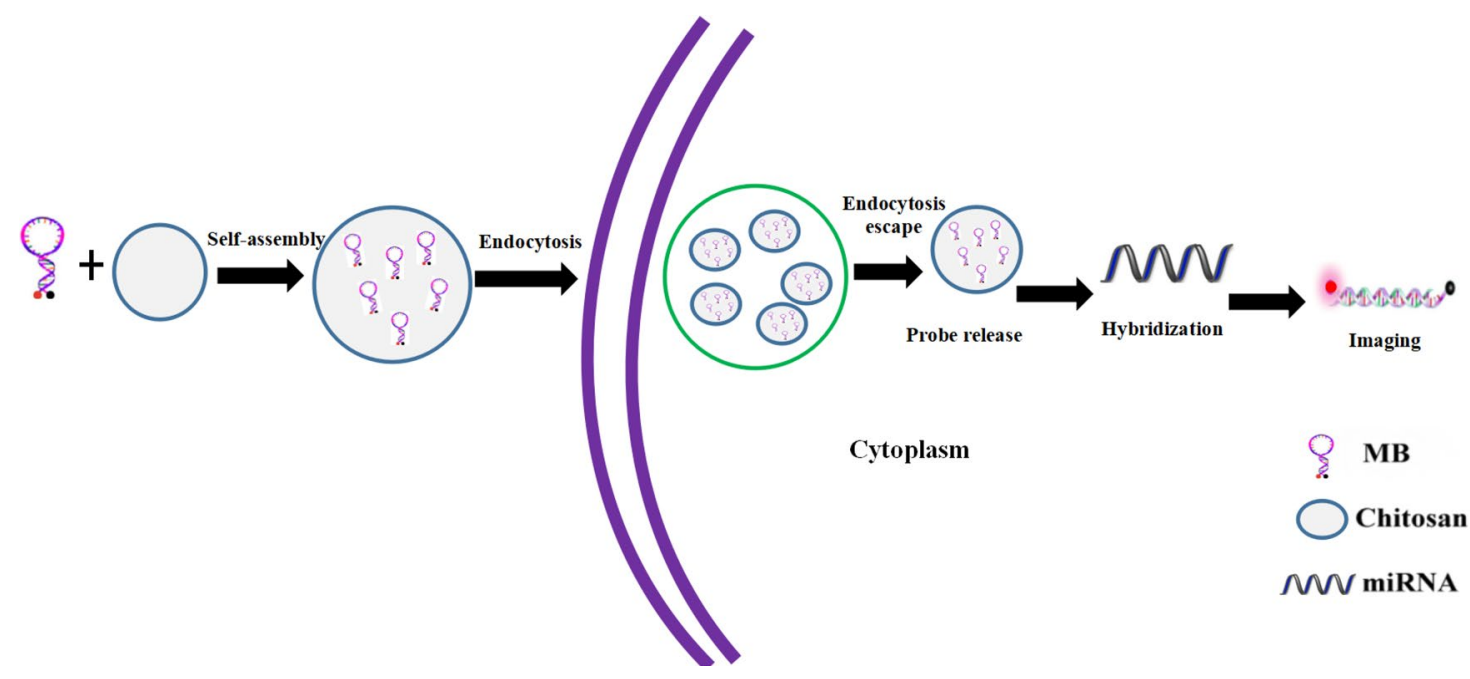

Fig. 1 Schematic showing the delivery of miR-155-5p MB into the cells via CS nanoparticles for the detection and imaging of miRNA 


\section{Materials and methods}

\section{Cell culture}

Human lung adenocarcinoma cells A549 and human small cell lung cancer cells (SCLC) H446 were purchased from American Type Culture Collection (Manassas, VA, USA). Human lung adenocarcinoma cells SPC-A1 were purchased from the Shanghai Institute of Cellular Biology. A549, SPC$\mathrm{A} 1$, and H446 lung cancer cell lines were cultured in an incubator with $5 \% \mathrm{CO}_{2}$ at $37^{\circ} \mathrm{C}$ using RPMI 1640 medium (Hyclone, Waltham, MA, USA) containing 10\% fetal bovine serum (Haoyang, Tianjin, China). According to the previous study (Yao et al. 2014), TICs (CD133 ${ }^{+} \mathrm{CD} 338^{+}$cells) were isolated from A549 cells as described previously and cultured in serum-free DMEM/F12 (Hyclone, Waltham, MA, USA) medium.

\section{Animal model establishment}

All in vivo experiments were approved by the Xinqiao Hospital Animal Care and Use Committee. Under aseptic experimental conditions, $1 \times 10^{6}$ A549 and H446 cells were subcutaneously injected into the left back or front of anesthetized 6-8-week nude mice to establish subcutaneous xenograft models of lung adenocarcinoma and small cell lung cancer. $1 \times 10^{6}$ A549 and H446 cells were injected via the tail veins to establish lung xenograft models. Thirty days following injection of lung cancer cells, subcutaneous transplanted tumors $\left(\mathrm{Vol} \sim 300 \mathrm{~mm}^{3}\right)$ and lung transplanted tumors appeared in all mice. Cre adenovirus (Hanbio, Shenzhen, China), with the titer of $5 \times 10^{9}$ PFU that can secrete Cre enzyme, was slowly instilled into the nasal cavity of the transgenic mice at the age of 8 weeks. These mice were sacrificed at weeks $4,6,8$, and 12 after instilling adenovirus. One side of the lung tissues in the transgenic mice at different disease stages and the lung xenograft models were resected, fixed in $4 \%$ paraformaldehyde (Boster, Wuhan, China), and sliced as $4 \mu \mathrm{m}$ paraffin sections for $\mathrm{HE}$ (hematoxylin-eosin) staining to observe the different pathological changes. The subcutaneous xenografts and lung xenografts from the other side were frozen in the liquid nitrogen for detecting miR-155-5p expression.

\section{Confocal microscopy}

Based on a previous study (Zhu et al. 2014), CS nanoparticles (Guanghan Hengyu, Sichuan, China), hsamiR-155-5p MB, mmu-miR-155-5p MB, or random sequence MB (RS MB) (Sangon, Shanghai, China) were mixed at the ratio of $\mathrm{Wcs} / \mathrm{W}_{\mathrm{MB}}=7: 1$ to synthesize the CS-MB nanoparticles. Hsa-miR-155-5p MB (5'Cy5-C + CAGCG-ACC + CCT + ATCA + CGAT + TAG
CATTAA-CGCT + GG-BHQ3-3') and Mmu-miR-155-5pMB $\left(5^{\prime}-\mathrm{Cy} 5-\underline{\mathrm{C}}+\mathrm{CAGCG}-\mathrm{ACC}+\mathrm{CCT}+\mathrm{ATCA}+\mathrm{CAAT}+\mathrm{TAG}\right.$ CAT TAA-CGCT + GG-BHQ3-3') were designed according to the miR-155-5p sequences. A random sequence $\mathrm{MB}$ ( $\mathrm{RS} \mathrm{MB}$ : 5'-Cy5-C+CAGCG$\mathrm{AC}+\mathrm{GCCA}+\mathrm{ATG}+\mathrm{ACC}+\mathrm{TTA}+\mathrm{AGCATTAA}-$ CGCT + GG-BHQ3-3') complementary to no known gene sequence was used as a negative control. The MB had a Cy5molecule attached to the 5 '-end and a black hole quencher3(BHQ3) attached to the 3 '-end. The underlining bases were the ones added to form a stem, and the $+\mathrm{N}$ represented the LNAs (locked nucleic acids) modified bases. All the synthetic oligodeoxynucleotides were purchased from Sangon Company (Shanghai, China). $1 \times 10^{4}$ A549, SPC-A1, H446, and TIC lung cancer cells were seeded in culture dishes for $24 \mathrm{~h}$. Subsequently, $200 \mu \mathrm{L}$ of mixed CS-MB nanoparticles were added into the culture dishes, and $100 \mu \mathrm{L}$ Opti-MEM ${ }^{\circledR}$ I reduced serum medium (Life Technologies, Waltham, MA, USA) was added to adjust the concentration; and the final concentration of MB was $200 \mathrm{nmol} / \mathrm{L}$. RS MB was used as a negative control. These cells were incubated in a $\mathrm{CO}_{2}$ incubator at $37{ }^{\circ} \mathrm{C}$ for $2 \mathrm{~h}$, followed by PBS washes. Then, the nuclei were stained with Hoechst 33342 (Beyotime, Shanghai, China) at $37{ }^{\circ} \mathrm{C}$ for $20 \mathrm{~min}$, followed by imaging using confocal microscopy (Leica TCS Confocal Microscope, Leica, Wetzlar, Germany). An equivalent of $1 \mathrm{~mL}$ cell lysate (Beyotime, Shanghai, China) was added into each culture dish for cell lysis. A volume of $100 \mathrm{ml}$ suspension was distributed into each of 6-wells of a 96-well plate, and the fluorescence intensity of Cy5 (excitation $649 \mathrm{~nm} / \mathrm{emission} 670 \mathrm{~nm}$ ) per well was detected by Varioskan Flash (Thermo, Waltham, MA, USA) in each group.

\section{Quantitative RT-PCR}

Total RNA of cells and tissues was extracted from A549, SPC-A1, H446 cells, and TICs, as well as subcutaneous xenografts, lung xenografts, and lung tissues of transgenic mice at different stages of the disease using RNAiso plus TRIzol reagent (TaKaRa, Japan). One microgram total RNA was used for cDNA synthesis,reverse transcription was performed with the PrimeScript RT reagent kit (Takara, Japan) at $37{ }^{\circ} \mathrm{C}$ for $15 \mathrm{~min}$ and $85^{\circ} \mathrm{C}$ for $5 \mathrm{~s}$. The changes in the expression of miR-155-5p in the four types of cells, xenografts, and transgenic mice lung tissues were detected using the SYBR PCR Master Mix reagent kits (TaKaRa, Japan). The PCR conditions for the miR-155 and U6 genes were: pre-denaturation at $95{ }^{\circ} \mathrm{C}$ for $30 \mathrm{~s}$, followed by 40 cycles of denaturation $95{ }^{\circ} \mathrm{C}$ for $5 \mathrm{~s}$ and $60^{\circ} \mathrm{C}$ for $34 \mathrm{~s}$. For each sample, three duplicated wells were used for miRNA detection. U6 served as the internal reference gene and hasmiR-155-5p or mmu-miR-155-5p as the target gene. The relative expression of miR-155-5p was evaluated using the $\Delta \Delta \mathrm{Ct}$ method. All the premier sequences were synthesized 
by RiboBio Company (Riobio, Guangzhou, China). The hsa-miR-155-5p, mmu-miR-155-5p, and U6 Primer Set catalog numbers' are MQPS0000685, MQPS0002476, and MQPS0000002, respectively.

\section{In vivo detection of miR-155-5p and imaging of the cancer cells}

After the mice were anesthetized by intraperitoneal injection of $4 \%$ chloral hydrate (Chron Chemicals, Chengdu, China), an equivalent of $100 \mu \mathrm{L}$ of CS-miR-155-5p MB or
CS-RS MB nanoparticles was injected via the tail veins. The final concentration of MB was $2 \mu \mathrm{M}$. There are two negative control groups, in the subcutaneous and lung xenograft nude mice models; A549 subcutaneous and lung xenografts models were used as the negative control groups after the injection of CS-RS MB; in the transgenic mice model, mice without intranasal inhalation of the adenovirus after the injection of CS-miR-155-5p MB were used as the negative control group. Depilatory cream was used for removing the black hair on the thorax of the transgenic mice for detection of Cy5 fluorescence signal. After $2 \mathrm{~h}$, the mice were
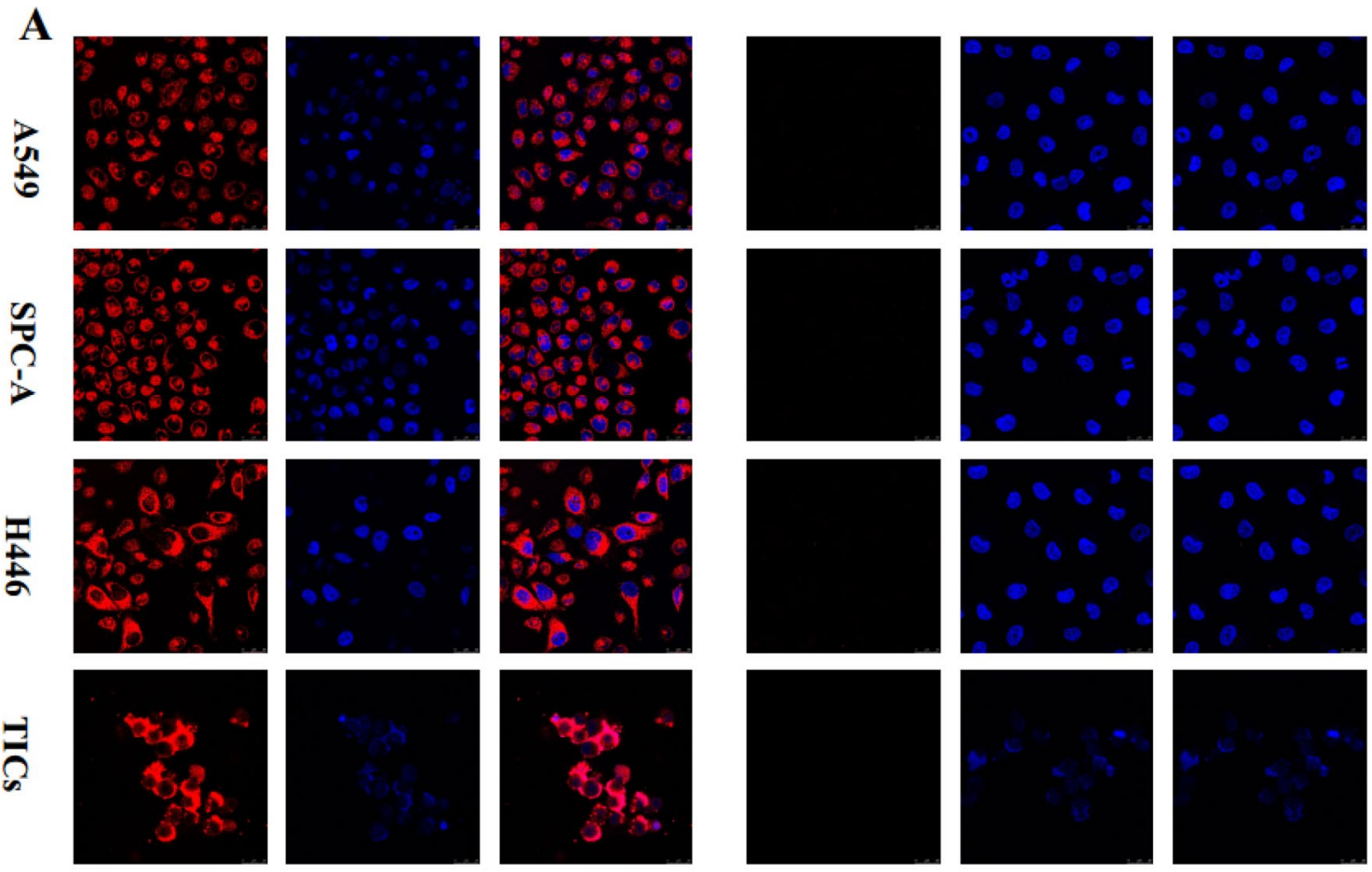

CS-miR-155 MB

Hoechst33342

Merge
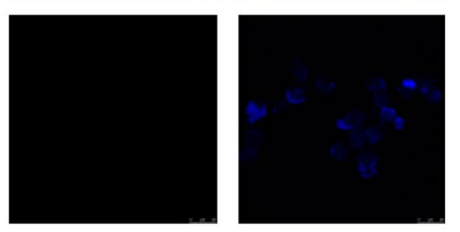

CS-RS MB

Hoechst33342

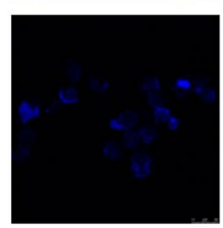

Merge

B

C
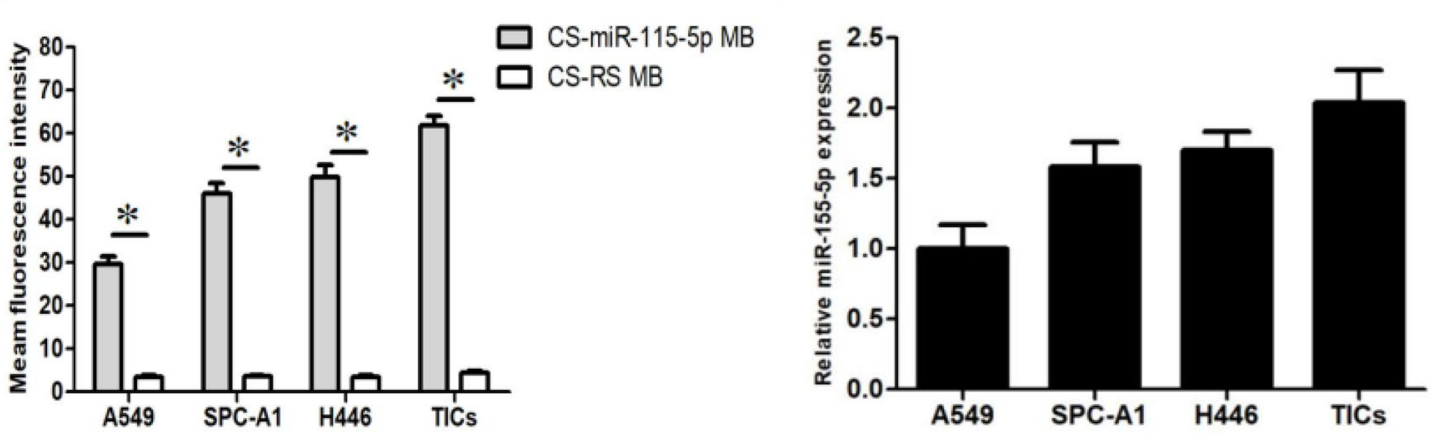

Fig. 2 Fluorescence imaging and detection in viable cancer cells. a Confocal microscopy imaging of the four cells after delivery of the miR-155-5p MB (red) by CS nanoparticles. RS MB was used as a negative control. The cell nucleuses were stained by Hoechst 33342

(blue). Scale bar $=25 \mu \mathrm{m}$. b Fluorescence intensity of Cy5 was measured after imaging $(n=6)(* p<0.05)$. c Relative miR-155-5p expression was detected in A549, SPC-A1, H446 cells, and TICs by qRTPCR 
placed on the in vivo imaging system (IVIS) platform, and the fluorescence signals from subcutaneous xenografts, lung xenografts, and transgenic mice lung tissues were imaged and detected by the IVIS Spectrum Imaging System (Caliper Life Sciences, Boston, USA). After in vivo imaging, the mice were sacrificed by dislocating the neck. The xenografts and lung tissues were removed and imaged again by the IVIS Spectrum Imaging System. Next, the frozen sections were prepared and then fixed in paraformaldehyde, followed by DAPI (Beyotime, Shanghai, China) staining of the nucleus. After that, the fluorescent images were photographed by confocal microscopy.

\section{Detection and imaging of miR-155-5p in the human lung cancer tissues}

This study was approved by the Medical Ethics Committee of Xinqiao Hospital (NO.2017004-011), and all participants were completely informed and signed the written informed consents. As described previously (Peng et al. 2005), three cases of fresh lung squamous cell carcinoma specimens and three cases of adenocarcinoma specimens were collected from the XinQiao Hospital of Army Medical University after confirmed by postoperative pathology. Frozen sections were prepared and fixed in ice acetone (Chron Chemicals, Chengdu, China) for $10 \mathrm{~min}$. Then, $50 \mu \mathrm{L}$ hsa-miR-155-5p $\mathrm{MB}$ or RS MB at $100 \mathrm{nmol} / \mathrm{L}$ was dropped to each section, followed by incubation at $37^{\circ} \mathrm{C}$ for $60 \mathrm{~min}$. Subsequently, the nuclei were stained by DAPI. The fluorescence signal of miR-155 in the lung tissues was detected by confocal microscopy; RS MB was used as the negative control.

\section{Statistical analysis}

An independent sample $t$ test was performed for comparison between the two groups, and one-way ANOVA was performed for comparison among multiple groups. $P<0.05$ was considered statistically significant. All data were represented as means \pm standard deviations.

\section{Results}

\section{Fluorescence imaging of cancer cells in vitro}

To determine whether the CS-MB probe could detect the level of miRNA expression in viable cells, their ability to detect target microRNAs was assessed. In vitro experiments
A

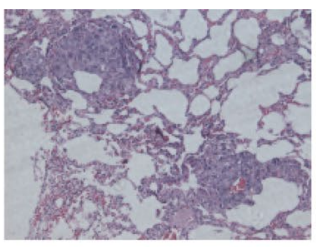

B

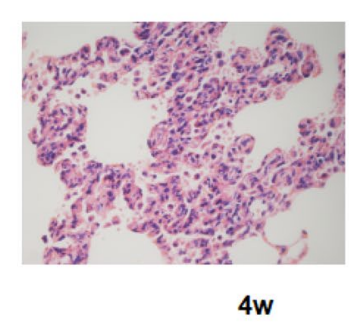

C

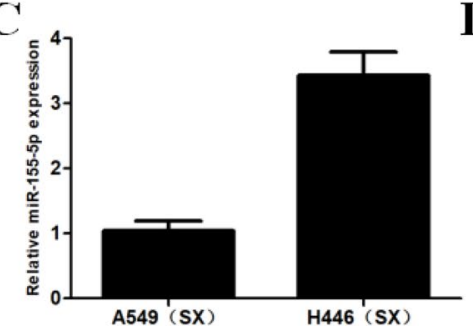

A549 (LX)

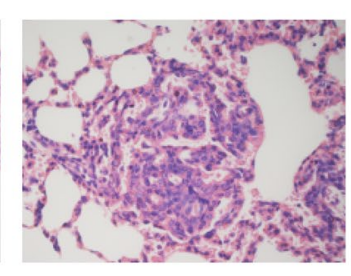

$6 w$

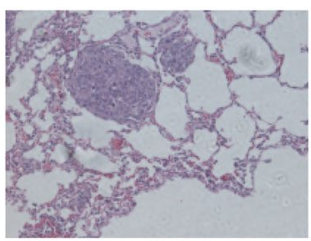

H446 (LX)

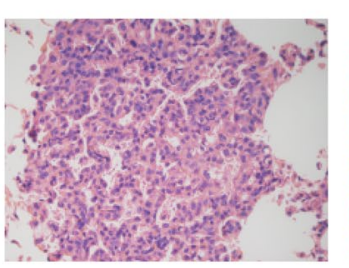

$8 w$

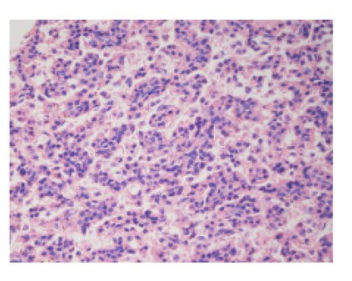

$12 w$
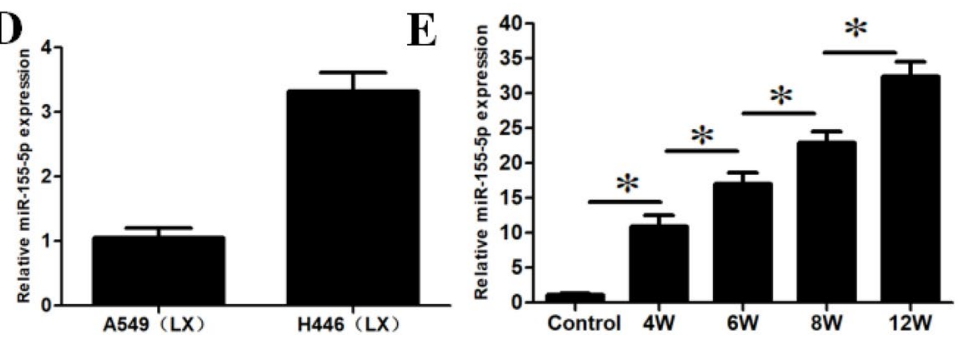

Fig. 3 Establishment of animal models and detection of miR-155-5p expression. a HE staining in A549 and H446 lung xenograft(LX) models $(\times 200)$. b HE staining at 4, 6, 8, and 12 weeks in transgenic mice models after instillation of adenovirus $(\times 400)$. $\mathbf{c}, \mathbf{d}$ miR-155-5p

expression in the subcutaneous xenografts (SX) and lung xenografts (LX) of nude mice models $(n=8)$. e miR-155-5p expression in transgenic mice at different disease stages $(n=8)\left({ }^{*} p<0.05\right)$ 


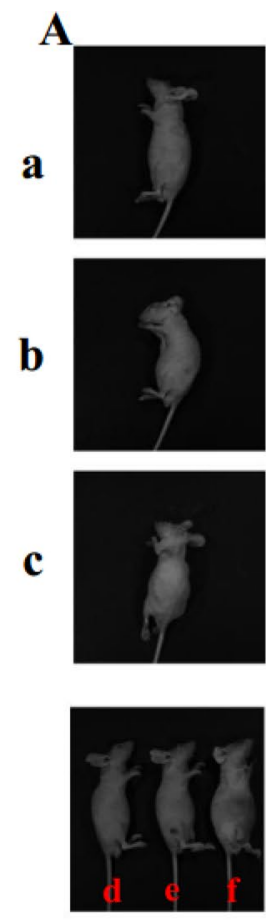

Brightfield
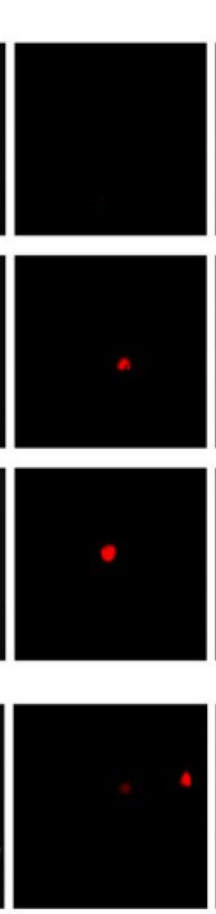

Fluorescence
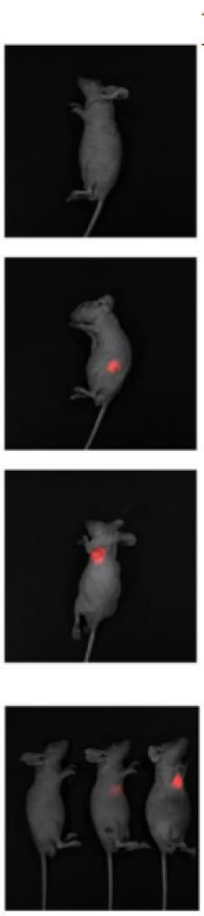

Merg
B
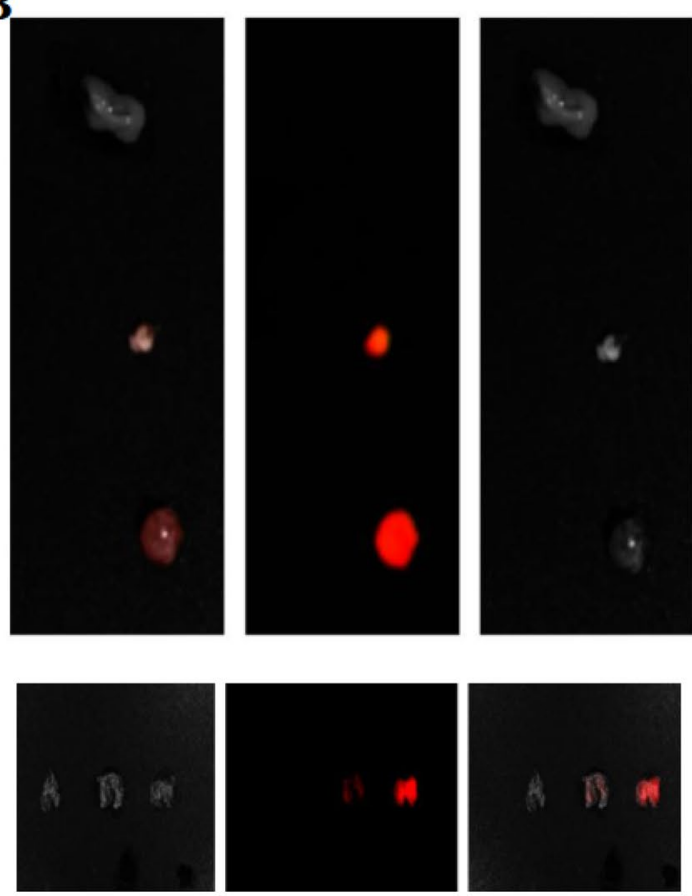

Brightfield

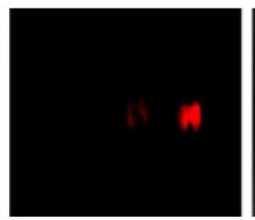

Fluorescence

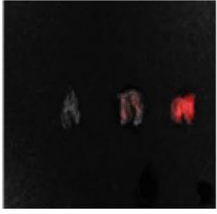

Merg

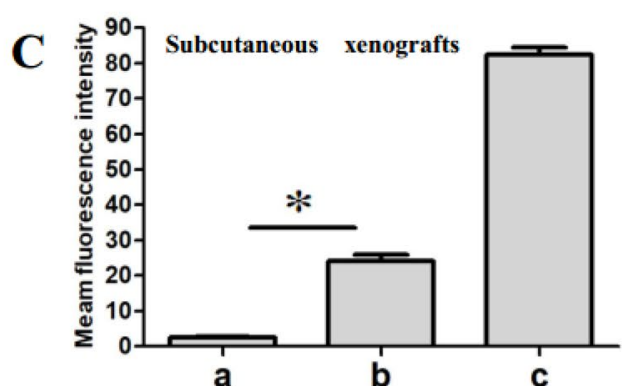

D Subcutaneous xenografts

a

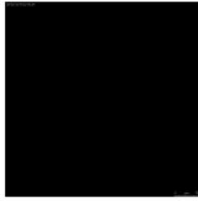

b

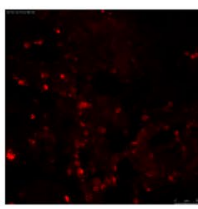

c

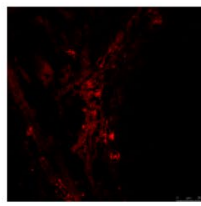

Fluorescence
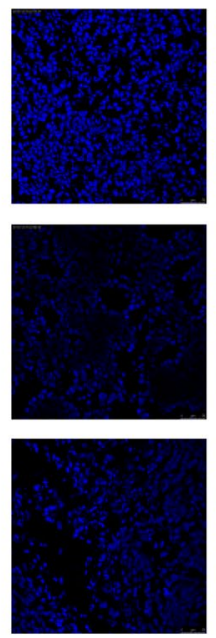

DAPI
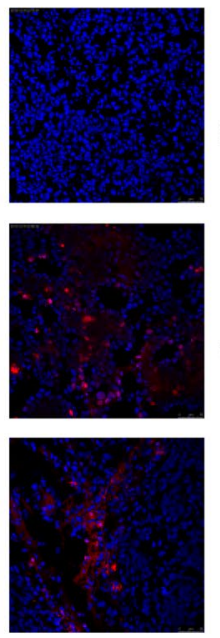

Merge

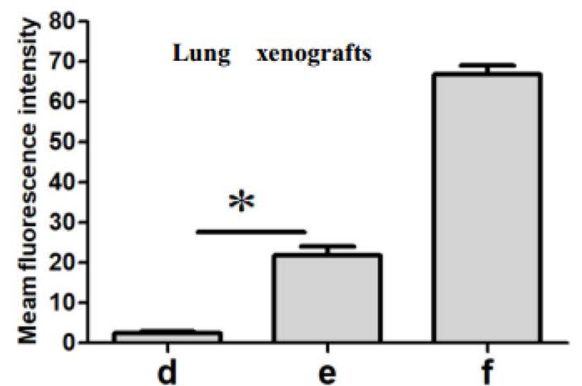

Lung xenografts
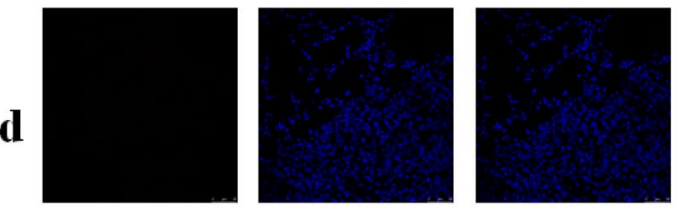

e
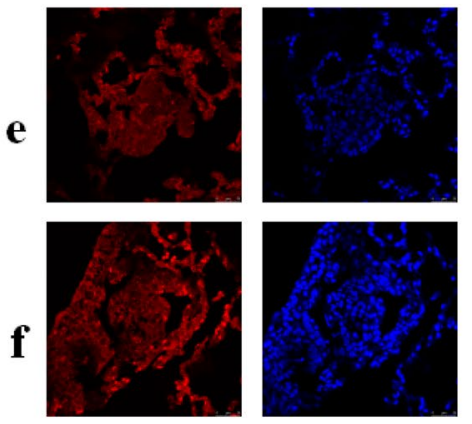

Fluorescence

DAPI
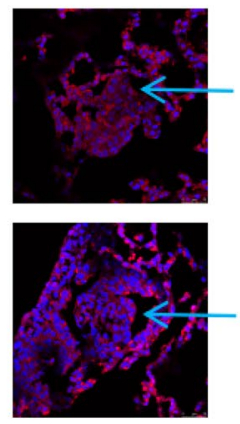

Merge 
४Fig. 4 In vivo identification of miR-155-5p and fluorescence imaging of cancer cells in xenografts models. a Imaging the subcutaneous and lung xenografts after injection of CS-MB via the tail veins. Subcutaneous xenografts model, a: A549 treated with CS-RS MB, b:A549 treated with CS-miR-155-5p MB, c: H446 treated with CS-miR155-5p MB. Lung xenografts model, d: A549 treated with CS-RS MB, e:A549 treated with CS-miR-155-5p MB, f: H446 treated with CS-miR-155-5p MB. Groups a and d were used as the negative controls. b Imaging the xenografts after removal. c Fluorescence intensity was analyzed after injection $(n=8)(* p<0.05)$. d Confocal microscopy imaging of the xenografts tissues after transfection with CS-miR155-5p MB or CS-RS MB (red). Cell nuclei were stained by DAPI (blue). Scale bar 25, $50 \mu \mathrm{m}$. Arrow: planting nodule and cancer cells

showed strong red fluorescent signals in the cytoplasm in each group of cells in the presence of CS-miR-155 MB, while only a few signals were detected in the nuclei (Fig. 2a). However, no significant red fluorescent signals were detected in the CS-RS MB group (Fig. 2b). Furthermore, the average fluorescence intensity of the 6 wells in each group was calculated. The mean fluorescence intensity of TICs was the strongest, followed by H446, SPC-A1, and A549 cells, and the fluorescence intensity was significantly increased in the presence of the CS-miR-155-5p MB compare with the presence of the CS-RS MB (Fig. 2c). Moreover, the fluorescence intensity trend of the four cells, after miR-155-5p MB was transfected using CS nanoparticles, was similar to the expression of miR-155-5p detected by qRT-PCR (Fig. 2c). Therefore, CS-MB probe could be used for detecting miR$155-5 p$ expression and imaging lung cancer cells.

\section{HE staining and miR-155-5p expression in xenografts and transgenic mice models}

HE staining was used to prove that lung xenograft model and different disease stages of transgenic mice were established, while qRT-PCR technology was used to detect the expression of miR-155-5p in lung cancer cells and animal models. HE staining revealed that lung adenocarcinoma cells A549 and small cell lung cancer cells H446 planting nodules were implanted in the lung tissues of nude mice (Fig. 3a), indicating successfully establishment of lung xenograft models. At 4, 6, 8, and 12 weeks after instillation of adenovirus, different disease processes of atypical hyperplasia, adenoma, carcinoma in situ, and lung adenocarcinoma could be observed in lung tissues of the transgenic mice (Fig. 3B), suggesting that lung cancer models of different disease stages were established successfully. qRT-PCR showed that miR-155-5p expressed in subcutaneous xenografts (SX) and lung xenografts (LX) of nude mice (Fig. 3c, d) as well as lung tissues of transgenic mice at different stages of the disease. In transgenic mice, miR155-5p expression increased significantly with the progression of lung cancer as compared to that at $4,6,8$, and 12 weeks (Fig. 3e), suggesting that miR-155-5p plays a major role in the occurrence and development of lung cancer, and can be used as a target for tracing, detection, and imaging.

\section{Fluorescence imaging of cancer cells in xenografts models}

The ability of CS-MB probe to detect miRNA in vivo was further investigated in xenografts models. The in vivo imaging showed fluorescent signals with varying intensities were detected in the subcutaneous xenograft and chests (Fig. 4a). After the tumor or lung tissues were removed, the imaging showed that the fluorescent signals were stronger in the H446 group than in the A546 group. The distribution of the fluorescent signals was found to be consistent with the size of subcutaneous xenografts (Fig. 4b). The fluorescence intensity was analyzed after injection using the Spectrum Living Image 4.0 software, and the results demonstrated that fluorescent signals in the H446 group with high miR155-5p expression were stronger than those in the A549 group. However, no fluorescent signals were detected in the CS-RS MB negative control group of the A549 SX and LX models (Fig. 4c). Interestingly, the fluorescence intensity trend was similar to the expression of miR-155-5p detected by qRT-PCR (Fig. 3c, d). The frozen sections were prepared using tumor tissues and lung tissues and examined by confocal microscopy, which showed that the fluorescent signals were from some tumor cells in the subcutaneous xenografts model. In lung xenografts model, the fluorescent signals were from some tumor cells and some alveolar epithelial cells. Compared to the A549 group, the fluorescent signals were brighter in the H446 group (Fig. 4d). Moreover, the intense fluorescent signals in the cytoplasm suggested that CS nanoparticles could be used as carriers to transport MB in vivo, thereby facilitating its entry into the cytoplasm to specifically bind to miR-155 and produce the signals.

\section{Fluorescence imaging cancer cells in transgenic mice models}

The ability of CS-MB nanoparticles to detect miR-155-5p at the different disease stages in the transgenic mice was further investigated. After CS-MB nanoparticles were injected via the tail veins using the same method, fluorescent signals of varying intensities could be detected in the lung tissues of transgenic mice (Fig. 5a). Also, the fluorescent signals were detected at different disease stages after removal of the lung tissue (Fig. 5b). Analysis of the fluorescence intensity showed an increasing trend with the progression of the disease (Fig. 5c). However, the fluorescence signals were not detected in the control group of mice without intranasal inhalation of the adenovirus. It 


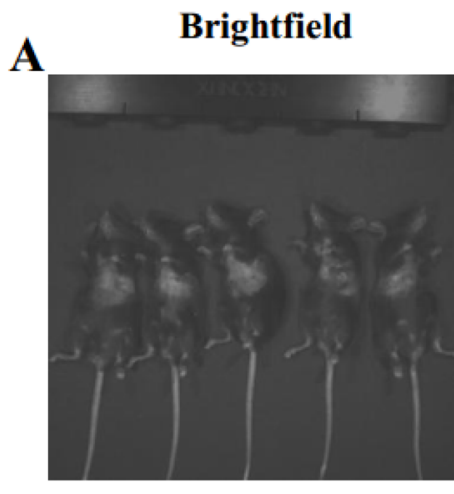

Fluorescence

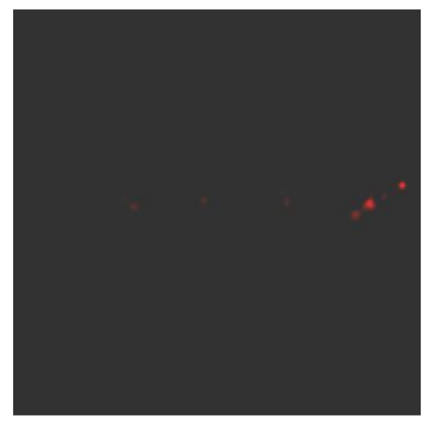

\section{Control,4w,6w,8w, $12 w$}

B
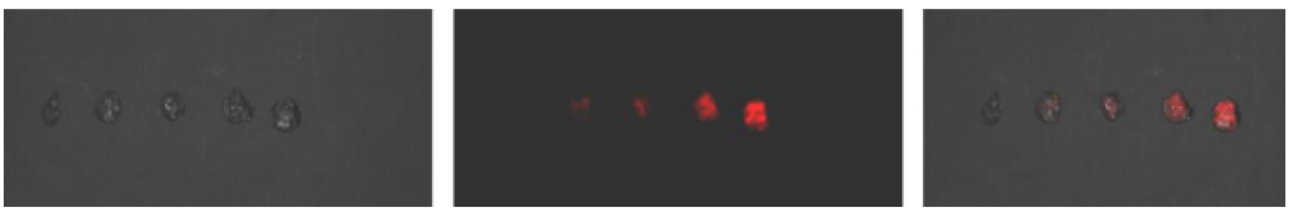

Control,4w,6w,8w, 12w

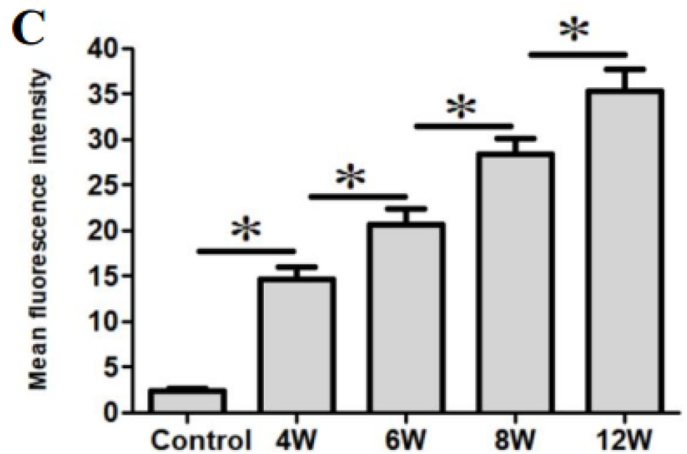

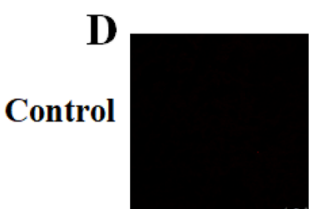
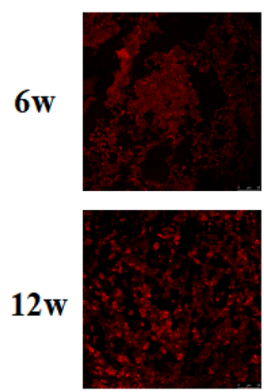

Fluorescence
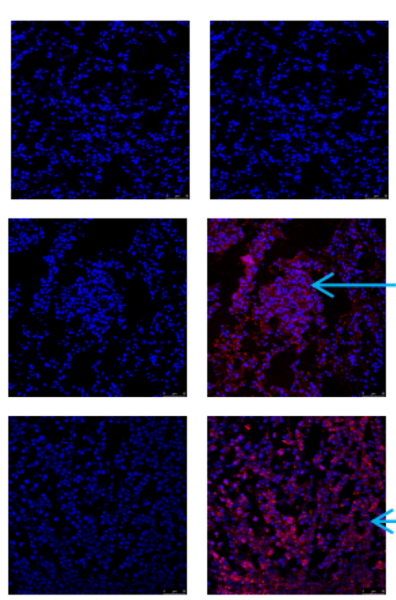

DAPI
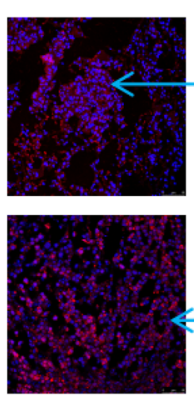

Merge
Fig. 5 In vivo identification of miR-155-5p and fluorescence imaging of cancer cells in transgenic mice at different stages of the disease. a Imaging the lung after injection of CS-miR-155-5p MB nanoparticles via the tail vein. Mice without intranasal inhalation of the adenovirus were used as the control group. b Imaging the lungs after removal. c Fluorescence intensity was analyzed after injection $(n=8)$ $\left({ }^{*} p<0.05\right)$. d Confocal microscopy imaging of the different pathological changes after transfection with CS-miR-155-5p MB. Cell nuclei were stained by DAPI (blue). Scale bar $50 \mu \mathrm{m}$. Arrow (1): atypical hyperplasia. Arrow (2): adenoma. Arrow(3): carcinoma in situ. Arrow (4): adenocarcinoma 
was similar to the expression of miR-155 detected by qRTPCR (Fig. 3e). Confocal microscopy of the frozen sections of the lung tissue showed that fluorescent signals were from the pleomorphic or cancer cells and some alveolar epithelial cells (Fig. 5d). The occurrence and development of lung cancer were dynamically monitored by detecting varying fluorescence intensities, which provided a novel technology for the early diagnosis of lung cancer.

\section{Fluorescence imaging and identification of miR-155 in human lung cancer tissues}

The ability of miR-155-5p MB to detect miR-155-5p in human lung cancer tissues was investigated further. After miR-155-5p MB was added to frozen lung cancer tissues, red fluorescent signals with different intensities could be detected in squamous and adenocarcinoma tissues. The majority of the red fluorescent signals were detected in the cytoplasm of the tumor. However, no significant fluorescent signals were detected in the negative RS MB group (Fig. 6). These findings suggested that miR-155-5p MB can bind to miR-155-5p in the tumor tissues, leading to fluorescent signals, which laid a foundation for subsequent preclinical study.

\section{Discussion}

Previous studies have demonstrated that miR-155 exerts a cancer-promoting effect in the occurrence and development of multiple cancers. miR-155 is not only overexpressed in several cancers such as breast, colorectal, gastric, and liver cancers, but also increased abnormally in the tissues and serum of the lung cancer patients, thereby miR-155 is a potential molecular marker for the early diagnosis of lung cancer, Moreover, lung cancer patients with high expression of miR-155 have a short survival time and poor prognosis (Liu et al. 2017; Zhu et al. 2018). MB technology can implement a convenient, rapid, and dynamic detection of miRNA. The detection of genes using MB is a highly sensitive and specific method, and can detect the difference of one base. This technology facilitates the detection of intracellular genetic mutations and changes in the expression of intracellular genes (Zhang et al. 2019a, b; Mahani et al. 2019).

Since MB is an oligonucleotide, it needs to be carried into live cells by ideal vectors. The optimal vectors not only protect the MB from degradation but also mediate target recognition of nucleic acid sequences in the cells, thereby facilitating the visualization study of live cells. CS has low toxicity and immunogenicity and can be obtained in a large quantity from the natural environment (Hejjaji et al. 2019). Due to its polycationic characteristics, chitosan can form complexes with negatively charged nucleic acids through electrostatic interactions, leading to the condensation and protection of nucleic acids. Chitosan has been regarded as a highly attractive biopolymer to deliver nucleic acids intracellularly and induce a transgenic response, so the CS nanoparticles were used as biomaterial carrier for MB delivery and miRNA detection in living cells (Santos-Carballal et al. 2018; Chuan et al. 2019).

Accumulating evidence has shown that TICs are the source of the occurrence, development, and recurrence of all tumors. Thus, finding and imaging TICs could provide a novel method for the early diagnosis of lung cancer (Peiffer et al. 2019). Therefore, in this study, lung adenocarcinoma cell lines A549 and SPC-A1, SCLC line H446, and TICs with high expression of miR-155-5p were used as models. After miR-155-5p MB was transfected with CS nanoparticles, the results showed that miR-155-5p MB could detect miR-155-5p and image the lung cancer living cells. Moreover, the fluorescence intensity of TICs with the highest expression of miR-155-5p was the maximal and can be easily detected. Recognized and imaged the TICs of lung cancer would be a method for the early diagnosis of lung cancer. Further analysis of the fluorescence intensity demonstrated that the fluorescence intensity of

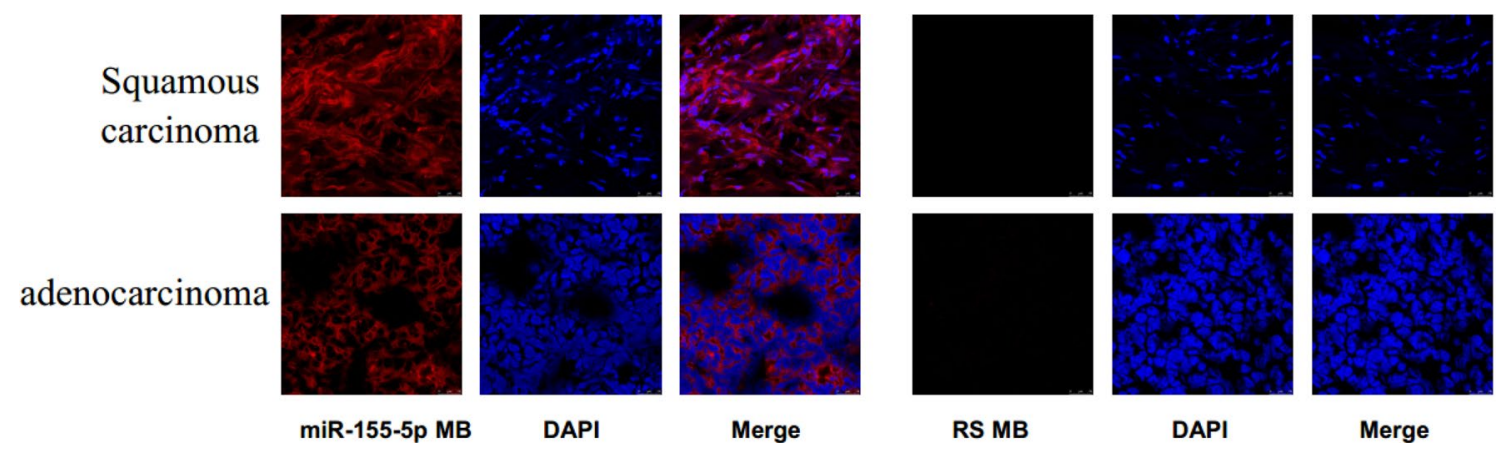

Fig. 6 Identification of miR-155-5p and fluorescence imaging of the cancer cells in human lung squamous carcinoma and adenocarcinoma tissues. RS MB was used as a negative control. Scale bar $50 \mu \mathrm{m}$ 
the four cell lines after transfection with MB was consistent with the expression of miR-155-5p as assessed by qRT-PCR. This phenomenon indicated that miR-155-5p MB designed in our study could dynamically monitor the changes in the expression of miR-155-5p in lung cancer living cells with high sensitivity. These findings demonstrated that miR-155-5p in living cells and TICs of lung cancer could be recognized and imaged at the cell level. Thus, the present study provided a new theoretical basis for searching lung cancer cells or tumor-initiating cells and applying them in the diagnosis of lung cancer.

At the animal level, after CS-MB nanoparticles were injected into xenograft and transgenic mouse models via the tail veins, strong or weak fluorescent signals could be detected in the tumor tissues. The endocytosis of CS-MB nanoparticles facilitates the entry and accumulation of MB in the tumor cells to identify microRNA, thus producing strong fluorescent signals, which could facilitate the early diagnosis of lung cancer by recognizing the highly expressed miR-155-5p and imaging the cancer cells. However, the fluorescent signals were detected in some alveolar epithelial cells, the specificity of the probe should be further investigated. In the other hand, dynamic monitoring of the occurrence and development of lung cancer based on the difference in the fluorescence intensity in transgenic mice models provided a novel method for the early diagnosis of the disease.

Several studies have demonstrated that miR-155 is highly expressed in the lung cancer tissues as compared to the normal lung tissues (Mohamed, et al. 2018). In the current study, tissue specimens from three cases of clinical lung squamous cell carcinoma and three cases of adenocarcinoma were used. The results showed that red fluorescent signals with different intensities could be detected after frozen lung cancer tissues were incubated with miR-155-5p $\mathrm{MB}$, indicating that miR-155-5p MB can bind to miR$155-5 \mathrm{p}$ in the tissues to produce fluorescent signals, which laid the foundation for subsequent preclinical studies.

In the current study, both in vivo and in vitro experiments showed that CS could be used as an ideal carrier of MB for entry into the cells. It can recognize the highly expressed miR-155-5p and image the cancer cells of TICs, lung cancer xenografts, and transgenic mice in the different stages of the disease. Furthermore, CS-MB nanoparticles could dynamically monitor the occurrence and development of lung cancer by detecting the difference in the fluorescence intensity. In addition, MB was used to primarily recognize and image miR-155-5p in human lung cancer tissues. Thus, this study provided a novel experimental basis for the early diagnosis of lung cancer and laid a foundation for subsequent preclinical studies. In addition, different MBs could be designed as targeted diagnostic probes to detect other highly expressed miRNAs in many different cancers or TICs.
Acknowledgements The work was supported by the Guizhou Provincial Natural Science Foundation (QKH-J[2020]1Y428), Gui Zhou Provincial Science and Technology Bureau Joint Foundation (Gui Zhou LH[2016]7196), Gui Zhou Provincial Health and Family Planning Commission Foundation (gzwjkj2017-1-002), the National Natural Science Foundation of China 81172070 and the Guizhou provincial High-level innovative talents plan. I would like to express my gratitude to my wife, Yi Guo, who helped me in writing this manuscript, especially language support.

Author contributions ZC and JS coordinated the research team. He conceived and designed the study with JS. HZ, CF, YG, and QZ carried out the experiments. The other authors who contributed to reagent preparation, material evaluation, data analysis, and structure elucidation are $\mathrm{LH}, \mathrm{DQ}, \mathrm{GC}, \mathrm{XP}$, and $\mathrm{JH}$.

\section{Compliance with ethical standards}

Conflict of interest The authors declare no conflict of interest.

Open Access This article is licensed under a Creative Commons Attribution 4.0 International License, which permits use, sharing, adaptation, distribution and reproduction in any medium or format, as long as you give appropriate credit to the original author(s) and the source, provide a link to the Creative Commons licence, and indicate if changes were made. The images or other third party material in this article are included in the article's Creative Commons licence, unless indicated otherwise in a credit line to the material. If material is not included in the article's Creative Commons licence and your intended use is not permitted by statutory regulation or exceeds the permitted use, you will need to obtain permission directly from the copyright holder. To view a copy of this licence, visit http://creativecommons.org/licenses/by/4.0/.

\section{References}

Cao Y, Tan YF, Wong YS, Liew MWJ, Venkatraman S (2019) Recent advances in chitosan-based carriers for gene delivery. Mar Drugs $17: 381$

Chuan D, Jin T, Fan R, Zhou L, Guo G (2019) Chitosan for gene delivery: methods for improvement and applications. Adv Colloid Interface Sci 268:25-38

De S, Lindner DJ, Coleman CJ, Wildey G, Dowlati A, Stark GR (2018) The FACT inhibitor CBL0137 synergizes with cisplatin in small-cell lung cancer by increasing NOTCH1 expression and targeting tumor-initiating cells. Cancer Res 78:2396-2406

Dong H, Ding L, Yan F, Ji H, Ju H (2011) The use of polyethylenimine-grafted graphene nanoribbon for cellular delivery of locked nucleic acid modified molecular beacon for recognition of microRNA. Biomaterials 32:3875-3882

DuPage M, Dooley AL, Jacks T (2009) Conditional mouse lung cancer models using adenoviral or lentiviral delivery of Cre recombinase. Nat Protoc 4:1064-1072

Hejjaji EMA, Smith AM, Morris GA (2019) Evaluation of the mucoadhesive properties of chitosan nanoparticles prepared using different chitosan to tripolyphosphate (CS:TPP) ratios. Int J Biol Macromol 120:1610-1617

Jaiswal S, Dutta PK, Kumar S, Koh J, Pandey S (2019) Methyl methacrylate modified chitosan: synthesis, characterization and application in drug and gene delivery. Carbohydr Polym 211:109-117 
Kang WJ, Cho YL, Chae JR, Lee JD, Choi KJ, Kim S (2011) Molecular beacon-based bioimaging of multiple microRNAs during myogenesis. Biomaterials 32:1915-1922

Kim KH et al (2015) Rapid, high-throughput, and direct molecular beacon delivery to human cancer cells using a nanowireincorporated and pneumatic pressure-driven microdevice. Small 11:6215-6224

Lingeshwar Reddy K, Prabhakar N, Rosenholm JM, Krishnan V (2018) Core-shell structures of upconversion nanocrystals coated with silica for near infrared light enabled optical imaging of cancer cells. Micromachines (Basel) 9:400

Lipińska J, Wawrzycki M, Jabłoński S (2019) Comparison of costs of hospitalization of patients with primary lung cancer after lobectomy with access through classic thoracotomy and VATS in the conditions of financing based on diagnosis-related groups. J Thorac Dis 11:3490-3495

Liu F, Song D, Wu Y, Liu X, Zhu J, Tang Y (2017) MiR-155 inhibits proliferation and invasion by directly targeting PDCD4 in nonsmall cell lung cancer. Thorac Cancer 8:613-619

Mahani M, Mousapour Z, Divsar F, Nomani A, Ju H (2019) A carbon dot and molecular beacon based fluorometric sensor for the cancer marker microRNA-21. Mikrochim Acta 186:132

Mohamed MA, Mohamed EI, El-Kaream SAA, Badawi MI, Darwish SH (2018) Underexpression of miR-486-5p but not overexpression of miR-155 is associated with lung cancer stages. Microrna $7: 120-127$

Moon SU et al (2019) Theragnosis by a miR-141-3p molecular beacon: simultaneous detection and sensitization of 5-fluorouracil resistant colorectal cancer cells through the activation of the TRIM13-associated apoptotic pathway. Chem Commun (Camb) 55:7466-7469

Moro M, Bertolini G, Pastorino U, Roz L, Sozzi G (2015) Combination treatment with all-trans retinoic acid prevents cisplatin-induced enrichment of CD133 + tumor-initiating cells and reveals heterogeneity of cancer stem cell compartment in lung cancer. J. Thorac. Oncol 10:1027-1036

Ost DE, Niu J, Zhao H, Grosu H, Giordano SH (2014) Quality gaps and comparative effectiveness in lung cancer staging and diagnosis. Chest 145:331-345

Peiffer DS et al (2019) DAXX suppresses tumor-initiating cells in estrogen receptor-positive breast cancer following endocrine therapy. Cancer Res 79:4965-4977

Peng XH et al (2005) Real-time detection of gene expression in cancer cells using molecular beacon imaging: new strategies for cancer research. Cancer Res 65:1909-1917

Rahmani S et al (2015) Novel chitosan based nanoparticles as gene delivery systems to cancerous and noncancerous cells. Int J Pharm 560:306-314

Santos-Carballal B, Fernández Fernández E, Goycoolea FM (2018) Chitosan in non-viral gene delivery: role of structure, characterization methods, and insights in cancer and rare diseases therapies. Polymers (Basel) 10:444

Shao C, Yang F, Qin Z, Jing X, Shu Y, Shen H (2019) The value of miR-155 as a biomarker for the diagnosis and prognosis of lung cancer: a systematic review with meta-analysis. BMC Cancer 19:1103-1112

Sutherland KD, Song JY, Kwon MC, Proost N, Zevenhoven J, Berns A (2014) Multiple cells-of-origin of mutant K-Ras-induced mouse lung adenocarcinoma. Proc Natl Acad Sci USA 111:4952-4957

Tutar L, Özgür A, Tutar Y (2018) Involvement of miRNAs and pseudogenes in cancer. Methods Mol Biol 1699:45-66

Wang JJ et al (2019a) Immunomodulatory protein from nectria haematococca induces apoptosis in lung cancer cells via the P53 pathway. Int J Mol Sci 20:5348

Wang Y et al (2019b) Vimentin expression in circulating tumor cells (CTCs) associated with liver metastases predicts poor progression-free survival in patients with advanced lung cancer. J Cancer Res Clin Oncol 145:2911-2920

Yao Q et al (2014) Monitoring microRNAs using a molecular beacon in CD133 +/CD338 + human lung adenocarcinoma-initiating A549 cells. Asian PacJ Cancer Prev 15:161-166

$\mathrm{Yu} \mathrm{T}$ et al (2016) KLF4 regulates adult lung tumor-initiating cells and represses K-Ras-mediated lung cancer. Cell Death Differ 23:207-215

Zhang R et al (2017) Multifunctional Molecular Beacon Micelles For Intracellular mRNA imaging and synergistic therapy in multidrugresistant cancer cells. Adv Funct Mater 27:1701027

Zhang Y, Zhao H, Zhang L (2018) Identification of the tumor-suppressive function of circular RNA FOXO3 in non-small cell lung cancer through sponging miR-155. Mol Med Rep 17:7692-7700

Zhang L et al (2019a) Identification and validation of novel circulating biomarkers for early diagnosis of lung cancer. Lung Cancer 135:130-137

Zhang P et al (2019b) A molecular beacon based surface-enhanced Raman scattering nanotag for noninvasive diagnosis of bladder cancer. J Biomed Nanotechnol 15:1589-1597

Zhu HZ et al (2014) Chitosan combined with molecular beacon for mir-155 detection and imaging in lung cancer. Molecules 19:14710-14722

Zhu HZ et al (2018) Identification and imaging of miR-155 in the early screening of lung cancer by targeted delivery of octreotideconjugated chitosan-molecular beacon nanoparticles. Drug Deliv 25:1974-1983

Publisher's Note Springer Nature remains neutral with regard to jurisdictional claims in published maps and institutional affiliations. 